\title{
风力机超声导波结冰探测方法的数值和试验研究
}

\author{
朱程香, 张玉雷, 朱春玲, 赵伟伟 \\ 南京航空航天大学飞行器先进设计技术国防重点学科实验室, 南京 210016 \\ ”联系人, Email: cxzhu@nuaa.edu.cn
}

收稿日期: 2016-06-11; 接受日期: 2016-10-14; 网络出版日期: 2016-11-09

国家自然科学基金(编号: 11402114)和国家重点基础研究发展计划(编号: 2015CB755800)资助项目

\begin{abstract}
摘要以结冰后的平面铝板为研究对象, 开展导波结冰探测方法的数值和试验研究. 采用两个压电元件粘贴 于铝板表面, 一个作为发生器, 另一个作为接收器, 并与铝板和冰层耦合, 建立分析模型, 采用数值模拟方法计 算发生器激发出的导波在结冰平面铝板中的传播特性, 分析导波的特征参数在不同频率条件下对冰层的敏感 度, 并建立结冰几何参数与导波传播特性之间的规律性关系. 建立导波结冰探测试验平台, 在不同的结冰长度 和结冰厚度条件下监测导波信号在铝板中的传播规律, 并与其在千净铝板中的传播特性进行对比, 分析结冰参 数对导波传播波形推移时间和峰值的影响规律, 验证数值方法的正确性. 计算和试验结果表明, 导波在结冰的 铝板中传播时, 波包到达时间会随着结冰厚度的增加而发生推迟, 且波包峰值随着结冰厚度的增加呈衰减趋 势; 而波包到达时间随着结冰长度的增加发生延迟, 但峰值不会随之改变。
\end{abstract}

关键词压电, 超声导波, 结冰探测, 模态分析, 结冰探测试验

PACS: $29.40 . \mathrm{WK}, 40.05 .+\mathrm{e}, 47.11 . \mathrm{Hj}, 47.50 . \mathrm{Cd}$

风力机结冰破坏了风力机叶片的气动外形, 改变 了风力机周围的流场特性 ${ }^{[1,2]}$, 降低风能利用率; 同时 不对称的结冰会引起风力机额外的过载和振动, 严重 时可能导致风力机停机. 因此监测风力机的结冰情况, 并采取合适的措施进行结冰防护对于保证风力机可 靠运行非常重要 ${ }^{[3]}$.

目前国外最广泛应用的结冰探测器是谐振式, 传 感器在一个固定频率点超声振动, 当有结冰附着时, 振动频率发生改变. 这种传感器可靠性较高, 但一般 采用外伸式安装, 影响了风力机的气动外形 ${ }^{[4]}$. 比较有 前景的结冰传感器 ${ }^{[5]}$ 包括了光纤式、平膜式、红外摄
像式等. 光纤式结冰传感器探头尺寸小, 灵敏度高, 便 于安装, 但是对水、油污、灰尘的干扰较为敏感. 平 膜式结冰传感器 ${ }^{[6]}$ 可以采用内埋式安装, 安装方便且 精度较高, 能区分冰和水及其他附着物, 缺点是需要 温度补偿, 最大可测冰厚度较小.

国内对结冰探测技术的研究比较滞后, 主要有哈 尔滨工程大学和华中科技大学进行了一些工作. 哈尔 滨工程大学研制了一种基于磁致伸缩原理的振管式 结冰传感器 ${ }^{[7]}$. 华中科技大学已成功研制出基于压电 效应的平膜式结冰传感器 ${ }^{[8]}$, 测量灵敏度可达 $0.1 \mathrm{~mm}$, 最大可测冰厚为 $2.0 \mathrm{~mm}$, 此外还在光纤式结冰传感器

引用格式: 朱程香, 张玉雷, 朱春玲, 等. 风力机超声导波结冰探测方法的数值和试验研究. 中国科学: 物理学 力学 天文学, 2016, 46: 124708 Zhu C X, Zhang Y L, Zhu C L, et al. Numerical and experimental study on icing detection of wind turbine blade based on ultrasonic guided wave (in Chinese). Sci Sin-Phys Mech Astron, 2016, 46: 124708, doi: 10.1360/SSPMA2016-00290 
的研究上取得了很大成功, 应用前景良好.

总体而言, 已有的结冰传感器大部分采用伸出式 安装方法, 影响了风力机附近的流场以及气动性能; 一些传感器只能探测到附近的结冰状况, 探测范围比 较小; 大多数传感器设计复杂, 体积重量大, 成本高.

本文针对国内外发展现状中存在的一些问题, 结 合超声波特有的传播距离长、信号稳定、不易衰减、 在特定的方向沿直线传播等优点 ${ }^{[9,10]}$, 将基于压电材 料的超声波检测技术应用于风力机叶片的结冰探测, 并开展了相关的数值和试验研究. 需要说明的是, 本 文将风力机叶片简化为平板, 针对平板表面的结冰探 测开展研究, 这主要是因为在无限均匀各向同性材料 中, 只存在两种类型的超声波, 即横波和纵波, 且两者 之间分别以各自的特性而不发生波形耦合, 虽然风力 机叶片为曲面, 导波在曲面中传播的横波和纵波受到 边界的制约以及边界处不断的模态转换, 但是可以通 过对称加载等方法有效降低其对主信号的干扰, 从而 提取出主信号, 关于信号分析及处理方法, 平板和曲 面是类似的.

\section{1 超声导波探测理论}

\section{1 超声导波在平板中的力学模型}

超声Lamb波是一种在厚度与激励波波长为相同 数量级的平板中由纵波和横波组合而成的特殊形式 的应力波, 它在不同厚度及激发频率下产生不同的传 播模式. 在平面应变假设下, 介质中有如下的应力位 移关系 ${ }^{[11]}$ :

$$
\begin{aligned}
& \tau_{y x}=\mu\left(\frac{\partial u_{y}}{\partial x}+\frac{\partial u_{x}}{\partial y}\right), \\
& \sigma_{y}=\lambda\left(\frac{\partial u_{x}}{\partial x}+\frac{\partial u_{y}}{\partial y}\right)+2 \mu \frac{\partial u_{y}}{\partial y},
\end{aligned}
$$

其中,

$$
\begin{aligned}
& u_{x}=\left[\begin{array}{l}
\left(A_{2} \mathrm{i} k \cos p y+B_{1} q \cos q y\right) \\
+\left(A_{1} \mathrm{i} k \sin p y-B_{2} q \sin q y\right)
\end{array}\right] \mathrm{e}^{\mathrm{i}(k x-w t)}, \\
& u_{y}=\left[\begin{array}{l}
-\left(A_{2} p \sin p y+B_{1} \mathrm{i} k \sin q y\right) \\
+\left(A_{1} p \cos p y-B_{2} \mathrm{i} k \cos q y\right)
\end{array}\right] \mathrm{e}^{\mathrm{i}(k x-w t)} .
\end{aligned}
$$

由铝板上下表面处的自由边界条件得

$$
\begin{aligned}
\frac{\left(k^{2}-q^{2}\right) \sin (q h)}{2 \mathrm{i} k p \sin (p h)} & =-\frac{2 \mu \mathrm{i} k q \cos (q h)}{\left(\lambda k^{2}+\lambda p^{2}+2 \mu p^{2}\right) \cos (p h)}, \\
\frac{\left(k^{2}-q^{2}\right) \cos (q h)}{2 \mathrm{i} k p \cos (p h)} & =\frac{2 \mu \mathrm{i} k q \sin (q h)}{\left(\lambda k^{2}+\lambda p^{2}+2 \mu p^{2}\right) \sin (p h)} .
\end{aligned}
$$

根据波速与材料属性的关系:

$$
\left(\lambda k^{2}+\lambda p^{2}+2 \mu p^{2}\right)=\mu\left(q^{2}-k^{2}\right)
$$

将(3)式化简为

$\frac{\tan (p h)}{\tan (q h)}=-\left[\frac{4 k^{2} p q}{\left(k^{2}-q^{2}\right)^{2}}\right]^{ \pm 1}$,

其中, +1 项对应对称模态, -1 项对应反对称模态.

$p=\sqrt{\frac{\omega^{2}}{c_{l}^{2}}-k^{2}} ; q=\sqrt{\frac{\omega^{2}}{c_{t}^{2}}-k^{2}}$,

其中 $c_{t}, c_{l}$ 分别为 $\mathrm{Lamb}$ 波的横波速度与纵波速度, 均为 已知常数, 波数 $k$ 数值上可表示为 $\omega / c_{p}, c_{p}$ 为 Lamb波传 播的相速度, $\omega=2 \pi f$.

为了精确求解方程(3), 本文利用二分法思想采用 Matlab计算软件求解方程并绘制结果 ${ }^{[12]}$, 如图1所示.

由图1可以看出, Lamb波具有两个特性: (1)多模 态性, 即随着频率的改变, Lamb波出现多个模态, 且频 率越大, 模态数越多; (2) 频散性, 即每一种模态的速度 随着频率的变化而改变.

用Lamb波进行结冰探测会因为信号中夹杂着许 多模态而变得复杂, 因此选择较小的加载频率, 从而 只产生 $\mathrm{S} 0$ 模态和 $\mathrm{A} 0$ 模态. 同时, $\mathrm{S} 0$ 模态的速度大于 $\mathrm{A} 0$ 模态, 信号特征参数对结冰信号更为敏感. 因此, 本文 采用 $\mathrm{S} 0$ 模态的特征变化检测分析表面结冰情况, 并选 择 $50 \mathrm{kHz}$ 作为加载信号的中心频率.

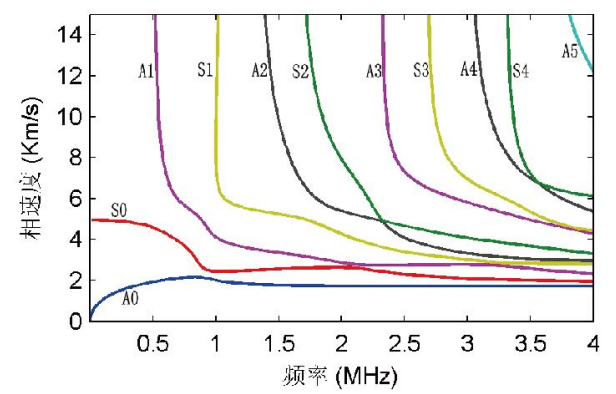

图 1 (网络版彩图)Lamb波频散曲线(相速度)

Figure1 (Color online) Lamb wave dispersion curve (phase velocity). 


\section{2 导波检测的物理模型}

微分或者积分过程过于复杂而无法获得解析解, 因此本文使用有限元法求解. 在计算过程中通过控制 单元类型, 网格划分密度等提高有限元的求解精度和 计算效率. 此外, 有限元也可以作为一种验证导波理 论的数值方法, 不仅可以验证导波频散曲线, 也可以 直观地观察导波的传播现象, 有助于分析结冰对超声 导波传播特性的影响. 导波为平面应力波, 在进行有 限元建模时候只需要考虑两个维度, 且二维问题分析 的效率较三维高. 本文采用的超声波发射接收示意图 如图2所示, 使用一个压电片作为发生器, 激发出 Lamb 波在铝板中传播, 经过结冰区域后波形及模态发生改 变, 之后信号被接收压电片接收. 将激励信号加载到 发生压电片上, 选择合适的距离作为接收端, 通过有 限元软件模拟接受点的位移响应作为接受压电片采 集到的结冰信号, 并和没有结冰时的信号进行对比, 进而判断结冰程度.

\section{2 结冰对探测信号的影响}

\section{1 对导波传播过程建模}

使用ANSYS有限元软件模拟Lamb波在不同结冰 情况下的探测信号, 对比结冰信号与健康信号, 观察 结冰信号随着结冰长度与厚度的变化规律. 厚度变 化时, 结冰长度统一为 $20 \mathrm{~mm}$, 长度变化时厚度统一 为 $1 \mathrm{~mm}$. 超声导波的传播是在固体中沿着长度方向传 播的, 因此, 为了简化模型本文模拟二维平面内 Lamb 波的传递情况, 模型如图3所示.

模型中, 铝板长 $400 \mathrm{~mm}$, 厚度为 $1 \mathrm{~mm}$, 距离原 点 $4 \mathrm{~mm}$ 处是激励压电片的位置, 冰型为明冰, 密度 为 $919.7 \mathrm{~g} / \mathrm{cm}^{3}$, 弹性模量为 $9.33 \times 10^{9} \mathrm{~Pa}$, 泊松比为 0.325 . 在软件中加载式(5)的激励函数 ${ }^{[13,14]}$

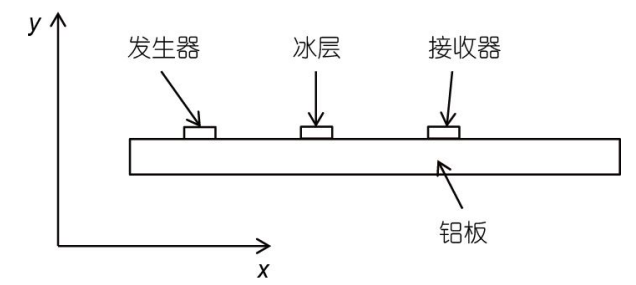

图 2 结冰探测物理模型

Figure 2 Physical model of icing detection.

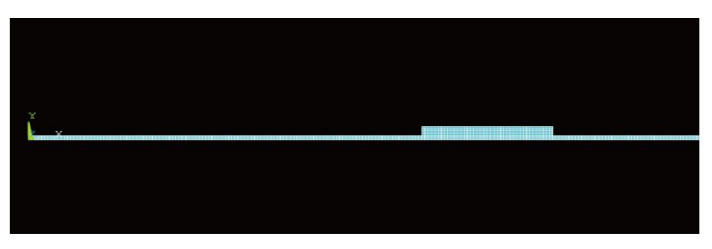

图 3 (网络版彩图)铝板表面结冰模型

Figure3 (Color online) Icing model of aluminum sheet.

$f(t)=0.5\left(1-\cos \frac{2 \pi f_{0} t}{n}\right) \sin \left(2 \pi f_{0} t\right)$,

其中 $f_{0}$ 为激励函数的中心频率, $n$ 为所加汉宁窗的个数. 本文中取中心频率为 $50 \mathrm{kHz}, n=5$. 在对模型进行加载 求解时选择纵波加载, 即压电片沿着坐标轴 $x$ 方向进位 移函数加载, 模型右端面约束, 加载完成后选择采集点 的 $U(X)$ 方向, 既采集点的 $X$ 方向位移量作为分析结果.

\section{2 数值模拟结果分析}

\subsection{1 冰厚度变化对导波传播特性的影响}

由于超声波在传播过程中遇到结冰会发生反射, 结冰处成为新的波源, 如若遇到边界又会产生边界反 射, 致使压电片接收到的信号不能单一的表示为结冰 的散射信号, 因此选择合适的位置作为信号接收点非 常关键. 前期的实验结果表明, 当发生器和接收器之 间的距离 $L$ 满足如式(8)的关系时, 可大大削弱边界反 射对探测信号的影响.

$\left\{\begin{array}{l}L / V_{\mathrm{S}}-L / V_{\mathrm{A}}>5 / f_{0}, \\ 2 S / V_{\mathrm{S}}>5 / f_{0},\end{array}\right.$

其中 $V_{\mathrm{S}} V_{\mathrm{A}}$ 分别为 $\mathrm{S}$ 模态和 $\mathrm{A}$ 模态的速度, $S$ 为接收压电 片与边界之间距离.

以 $x=250 \mathrm{~mm}$ 处上表面的节点为数据分析点. 选择 时间历程后处理器, 观察接收器对应位置处位移随时 间的变化关系, 如图4所示.

图4为Lamb波通过铝板表面的传播特性, 取相距5 $\mathrm{mm}$ 的两点 $n$ 和 $n+1$, 计算在 $50 \mathrm{kHz}$ 下 $\mathrm{S} 0$ 模态的群速度, 即 $\mathrm{S} 0$ 模态峰值处对应的到达时间, 如图 5 所示. 计算结 果为相距 $5 \mathrm{~mm}$ 两点 $\mathrm{S} 0$ 模态峰值到达时间相差 $1.027 \mu \mathrm{s}$, 由此可计算出此模型中 $S 0$ 模态的群速度为 $4868 \mathrm{~m} / \mathrm{s}$. 采用相同的模拟方法, 分别计算铝板表面结冰厚度为 $0.5,1,1.5,2,2.5,3,3.5,4,4.5,5 \mathrm{~mm}$ 时的Lamb波传播 特性, 如图6和7所示. 


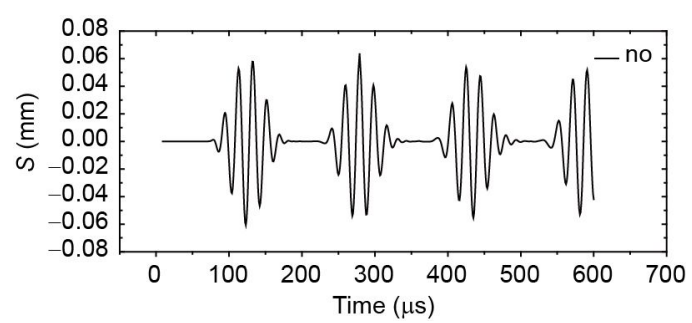

图 4 (网络版彩图) $x$ 处随时间的位移变化

Figure 4 (Color online) Displacement of $x$ with different time.

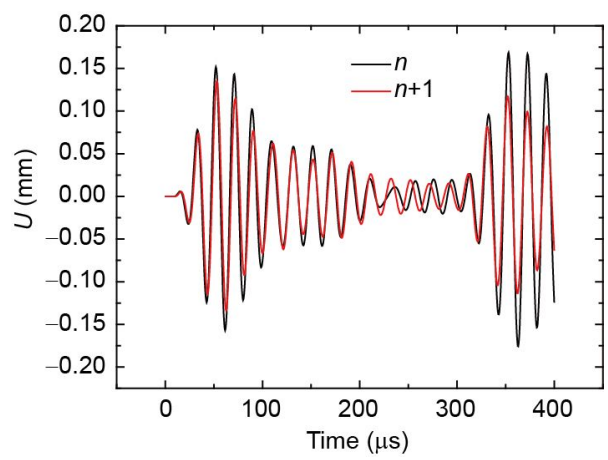

图 5 (网络版彩图)相距 $5 \mathrm{~mm}$ 的不同两点的时间推移

Figure 5 (Color online) Relationship of displacement and time of two different points with distance of $5 \mathrm{~mm}$.

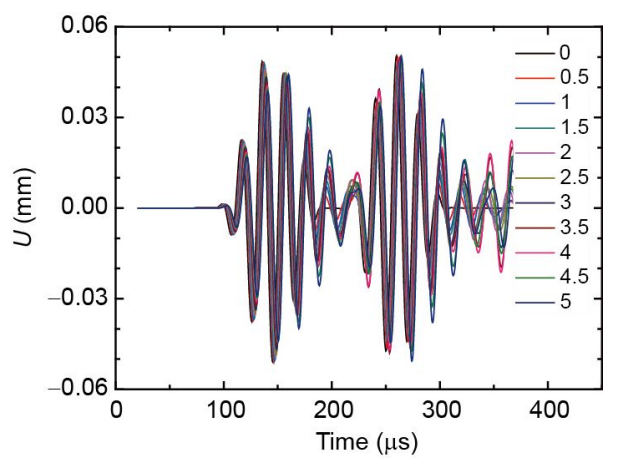

图 6 (网络版彩图)不同结冰厚度对Lamb波传播特性的影 响

Figure 6 (Color online) The influence of different ice thickness on the propagation characteristics of Lamb wave.

图6中黑色曲线(标识为: no) 为没有结冰时 $U(X)$ 的值, 其他为铝板表面覆冰后 Lamb波在 $X$ 方向的位移. 从峰值看, Lamb波在无冰铝板中传播的峰值明显比 有结冰时的峰值大. 图7为 $\mathrm{S} 0$ 模态峰值放大图, 没有结 冰时峰值为 0.04844 , 结冰厚度为 $1 \mathrm{~mm}$ 时峰值下降到 0.04819 , 下降了 $1.46 \%$. 随着结冰厚度的增加, 峰值逐 渐下降. 这是由于结冰的存在减弱了超声波的能量,
使得采集点的波峰值变小. 从波峰出现的时间来看, 没有结冰时, Lamb波波峰出现在 $0.1355 \times 10^{-3} \mathrm{~s}$, 当出现 $1 \mathrm{~mm}$ 后的结冰时, 波峰出现时间变为 $0.13700 \times 10^{-3} \mathrm{~s}$, 推迟了 $1.11 \%$. 从表 1 中可以看出随着结冰厚度的增加, 波峰到达时间呈现推迟的趋势, 但是这种变化比较细 微, 不易观察.

\subsection{2 结冰长度对导波传播特性的影响}

采用本文的有限元模拟方法, 对不同结冰长度下 导波的传播特性进行数值模拟, 结冰长度范围为 10 $\mathrm{mm}$ 到 $100 \mathrm{~mm}$, 每隔 $10 \mathrm{~mm}$ 计算一次. 得到不同结冰长 度下超声波的传播特性, 如图8和图9所示.

图9中黑色曲线为没有结冰情况下的Lamb波传播 特性, 其他为不同结冰长度下的Lamb波传播特性, 从 峰值看, 结冰后峰值比结冰前小, 并且随着结冰长度的 增加, 峰值数值基本保持不变, 这主要是受到模态转 换的影响. 结冰厚度与长度对导波传播的影响是不一 样的，并且波包发生时间推移的机理也是不同的，冰

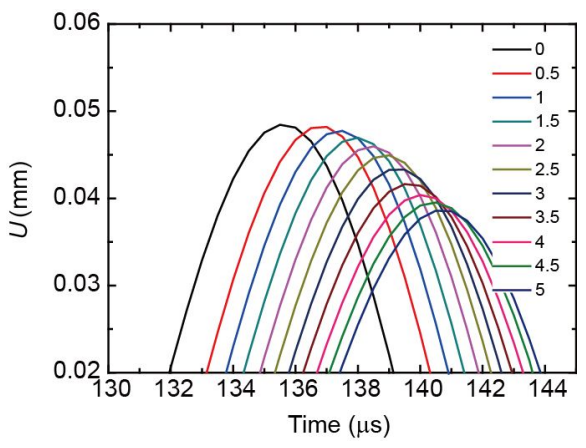

图 7 (网络版彩图)Lamb波峰值处的局部放大图

Figure 7 (Color online) Local display of Lamb wave crest value.

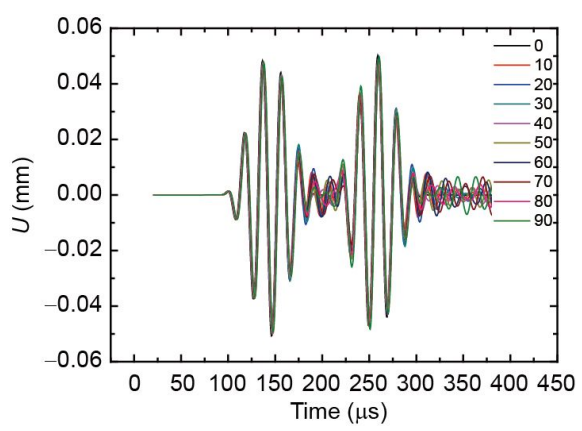

图 8 (网络版彩图)不同结冰长度对Lamb波传播特性的影 响

Figure 8 (Color online) The influence of different ice length on the propagation characteristics of Lamb wave. 
表 1 不同结冰厚度的模拟数值对比

Table 1 Numerical simulation of different icing thickness

\begin{tabular}{ccccc}
\hline 冰厚 $(\mathrm{mm})$ & 峰值时间 $(\mu \mathrm{s})$ & 变化幅度 $(\%)$ & 峰值 $(\mathrm{mm})$ & 变化幅度 $(\%)$ \\
\hline 0.0 & $0.13550 \times 10^{-03}$ & & 0.04844 & 0.52 \\
0.5 & $0.13650 \times 10^{-03}$ & 0.74 & 0.04819 & 1.46 \\
1.0 & $0.13700 \times 10^{-03}$ & 1.11 & 0.04774 & 3.08 \\
1.5 & $0.13750 \times 10^{-03}$ & 1.48 & 0.04695 & 5.12 \\
2.0 & $0.13800 \times 10^{-03}$ & 1.85 & 0.04596 & 7.24 \\
2.5 & $0.13850 \times 10^{-03}$ & 2.21 & 0.04493 & 10.61 \\
3.0 & $0.13900 \times 10^{-03}$ & 2.59 & 0.04330 & 14.07 \\
3.5 & $0.13950 \times 10^{-03}$ & 2.95 & 0.04163 & 16.64 \\
4.0 & $0.14000 \times 10^{-03}$ & 3.32 & 0.04038 & 18.42 \\
4.5 & $0.14050 \times 10^{-03}$ & 3.69 & 0.03952 & 0.03858 \\
5.0 & $0.14100 \times 10^{-03}$ & 4.06 & & 20.36 \\
\hline
\end{tabular}

表 2 不同结冰长度的数值模拟结果

Table 2 Results of numerical simulation of different icing lengths

\begin{tabular}{ccccc}
\hline 长度 $(\mathrm{mm})$ & 峰值时间 $(\mu \mathrm{s})$ & 变化幅度 $(\%)$ & 峰值 $(\mathrm{mm})$ & 0.04844 \\
\hline 0 & $0.13550 \times 10^{-03}$ & & 0.04715 & 2.66 \\
10 & $0.13659 \times 10^{-03}$ & 0.80 & 0.04725 & 2.45 \\
20 & $0.13680 \times 10^{-03}$ & 0.95 & 0.04719 & 2.58 \\
30 & $0.13694 \times 10^{-03}$ & 1.03 & 0.04726 & 2.43 \\
40 & $0.13706 \times 10^{-03}$ & 1.15 & 0.04723 & 2.49 \\
50 & $0.13726 \times 10^{-03}$ & 1.29 & 0.04720 & 2.56 \\
60 & $0.13737 \times 10^{-03}$ & 1.38 & 0.04718 & 2.60 \\
70 & $0.13772 \times 10^{-03}$ & 1.64 & 0.04708 & 2.80 \\
80 & $0.13788 \times 10^{-03}$ & 1.76 & 0.04718 & 2.60 \\
90 & $0.13804 \times 10^{-03}$ & 1.87 & & \\
\hline
\end{tabular}

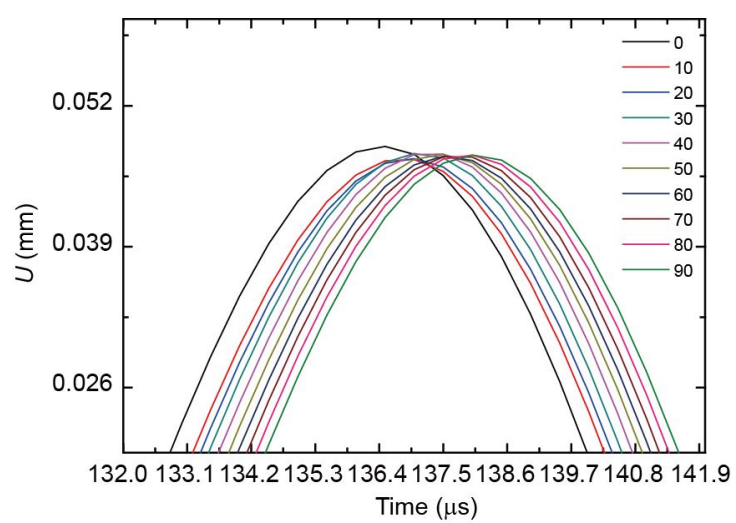

图 9 (网络版彩图)Lamb波峰值处的局部放大图

Figure 9 (Color online) Local display of Lamb wave crest value.

厚对模态时间推移是由于其对群速度的影响而引起 的, 而冰长度的影响是由于Lamb波穿过结冰区的距离 发生变化导致的.

\section{3 结冰对导波传播特性影响试验}

\section{1 结冰探测试验台}

根据超声波结冰探测原理搭建实验台. 采用的 仪器包括泰克AFG3022C 型信号发生器, T\&C Power Conversion公司生产的AG1020功率放大器, 阿尔泰公 司的PXI8502S型数据采集卡,此外本文的试验控制集 成在Labview平台上编程形成的采集程序, 具有控制方 便、采集简单的特点. 结冰探测实验台的原理示意图 如图10(a)所示, 实物图如图10(b)所示. 试验模型与数 值模拟模型保持一致, 通过发生传感器激发的Lamb波 在结冰的平面铝板传播, 并被接收传感器采集, 通过对 比试验测得的结冰前后超声波信号的 $\mathrm{S} 0$ 模态差异, 探 索结冰几何参数与导波传播特性之间的规律性关系. 本实验的实验模型是长 $400 \mathrm{~mm}$, 宽 $200 \mathrm{~mm}$, 厚 $1 \mathrm{~mm}$ 的 

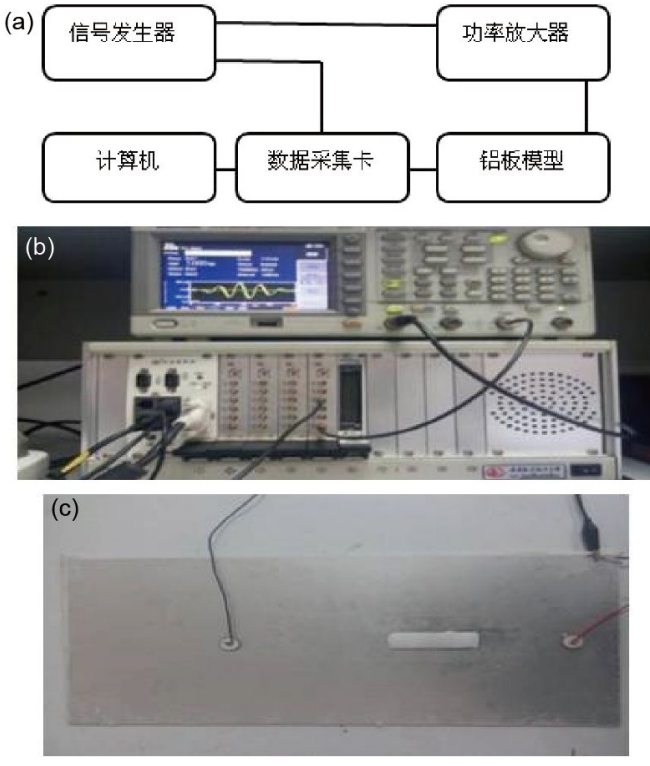

图 10 (网络版彩图)(a) 实验连接顺序图; (b) 信号发生器与 数据采集卡; (c) 结冰的平面铝板

Figure 10 (Color online) (a) Experimental connection sequence diagram; (b) signal generator and data acquisition card; (c) ice plane.

光滑平面铝板, 图10(c)所示, 激励压电片位于距离边 界 $50 \mathrm{~mm}$ 位置处, 接收压电片位于边界 $100 \mathrm{~mm}$ 处, 压 电片的规格为两个大小一致的 $\Phi 15 \times 2$ 的 $\mathrm{P} 4$ 压电片 ${ }^{[15]}$.

\section{2 数据采集}

实验中是按照采集卡设置的采样频率 $20 \mathrm{MHz}$ 进 行采样, 采样点数为 4096 个, 采集一个点需要 $0.05 \mu \mathrm{s}$, 采集完整个信号共需要时长为 $204.8 \mu \mathrm{s}$, 第一个采样点 为激励波传播到采集点的第一次位移. Lamb波在光滑 平面传播时波能量随着传播距离的增加逐渐衰弱, 为 了采集到的信号便于分析计算, 也为了能够实时观察 结冰引起的信号变化, 本文采用功率放大器对输入之 前的信号进行功率放大. 采集信号过程中, 通过功率 放大器将导播的幅值稳定在合适的位置后保持功率
不变, 观察在不同结冰厚度下超声波中 $\mathrm{S} 0$ 模态的传播 特性. 依次采集四组不同结冰厚度的信号, 观察结果.

\section{3 实验结果分析}

将采集到的健康信号与不同结冰厚度的结冰信 号进行对比, 分析结冰前后 S0模态的变化情况. 结果 如图11和12所示.

同样观察导波 $\mathrm{S} 0$ 模态的变化,表面没有结冰时,在 第 $136.6 \mu \mathrm{s}$ 时 $\mathrm{S} 0$ 模态波包的幅值达到本次实验的最高 值 $738 \mathrm{mV}$, 当结构表面出现 $1 \mathrm{~mm}$ 厚的结冰时, $\mathrm{S} 0$ 模态 波包的峰值点延迟了 $0.36 \%$, 随着结冰厚度的增加, S0 模态波包出现依次延迟的趋势, 但是变化细微. 随着 结冰厚度的增加, $\mathrm{S} 0$ 模态波包峰值点呈现明显的衰减 趋势, 这也是由于结冰厚度的增加, 对超声波的衰减越 厉害所造成的, 如图12所示. 造成这种变化是由于导

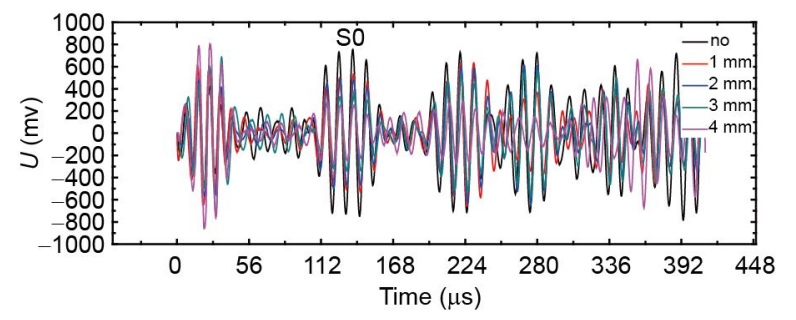

图 11 (网络版彩图)不同结冰厚度传感器处的位移

Figure 11 (Color online) Displacement of the same ice thickness sensor.

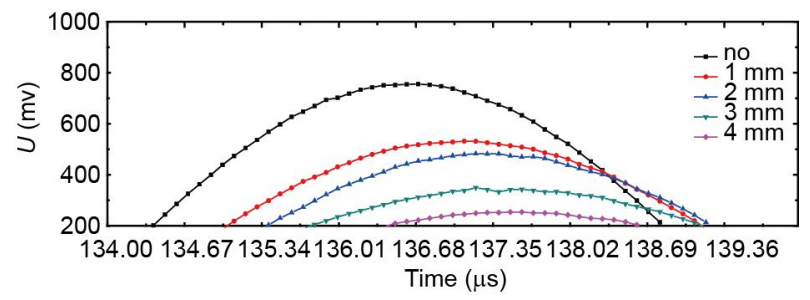

图 12 (网络版彩图) S0 模态处峰值的局部显示

Figure 12 (Color online) Local display of the peak value of S0 mode.

表 3 不同结冰长度的探测信号数据对比

Table 3 Comparison of detection signal data of different ice thickness

\begin{tabular}{ccccc}
\hline 结冰厚度 $(\mathrm{mm})$ & 峰值时间 $(\mu \mathrm{s})$ & 变化幅度 $(\%)$ & 电压峰值 $(\mu \mathrm{V})$ & 变化幅度 $(\%)$ \\
\hline 0 & 136.6 & - & 738.54124 & - \\
1 & 137.1 & 0.36 & 588.92619 & 20.1 \\
2 & 137.3 & 0.51 & 452.28396 & 38.7 \\
3 & 137.5 & 0.66 & 313.92263 & 60.4 \\
4 & 137.7 & 0.81 & 214.94525 & 71.6 \\
\hline
\end{tabular}


波遇到结冰时能量损失, 致使模态波峰值降低. 峰值 由于是通过功率放大器调节, 将功率放大到合适的位 置即结冰发生时的振幅衰减幅度和数值模拟一致时, 比较其他结冰厚度的变化幅度.

最后, 将试验结果和数值计算结果进行了对比, 如图13和14所示, 可以看出两者基本吻合, 验证了本 文数值方法的正确性. 但是两者也存在差异, 造成误 差的原因可能有以下几方面:

1)模拟计算没有对导波的反射, 映射进行模拟;

2)模拟时候激励函数通过加载方式之间作用到铝 板模型左端面, 对压电片的具体参数并没有考虑;

3)实验中的影响因素比较多, 首先, 外界振动引起 的干扰, 其次实验是在冷环境中进行, 而模拟高空真 实结冰环境存在差异.

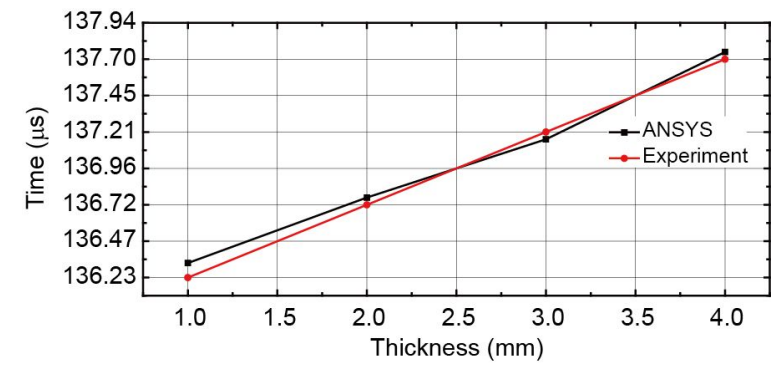

图 13 (网络版彩图) 模拟结果与数值结果的峰值点时间推 移对比

Figure 13 (Color online) Comparison of simulation results between the numerical results and the experimental results.

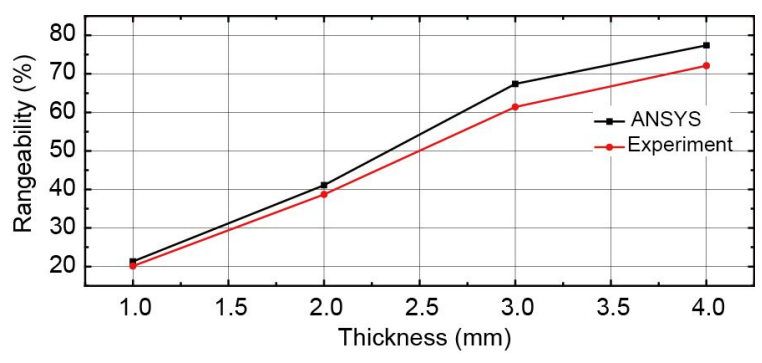

图 14 (网络版彩图)模拟结果与实验结果的峰值点变化幅 度对比

Figure 14 (Color online) Comparison of simulation results between the numerical results and the experimental results.

\section{4 结论}

本文建立了结冰冰层-压电元件-平面铝板耦合的 有限元分析模型, 采用数值模拟方法, 计算并对比分 析了结冰前后 Lamb波传播特性的差异, 验证了 Lamb 波用于结冰探测的可行性. 通过计算不同结冰长度和 厚度条件, Lamb波在铝板中的传播特性, 获得结冰长 度和厚度条件对Lamb波峰值出现的时间延迟和幅值 的影响规律. 建立的超声导波结冰探测试验台实现了 结冰条件的近似模拟和超声导波传播特性的准确监 测, 试验结果验证了数值模拟方法的正确性和本文建 立的结冰长度和厚度与传播特性之间规律的正确性. 结果表明, 随着结冰厚度的增加, $\mathrm{S} 0$ 模态波包的出现 时间发生延迟, 同时, 波包的峰值呈现逐渐衰减的趋 势; 随着结冰长度的增长, $\mathrm{S} 0$ 模态波包到达时间也出 现依次延迟, 但波包峰值变化不大.

\section{参考文献}

1 Lee S, Bragg M B. Investigation of factors affecting iced-airfoil aerodynamics. J Aircraft, 2003, 40: 499-508

2 National Transportation Safety Board. NTSB Safety Alerts: Aircraft Inflight Icing. SA-014, 2008

3 Han Y, Palacios J. Analytical and experimental determination of airfoil performance degradation due to ice accretion. In: Proceedings of 4th AIAA Atmospheric and Space Environments Conference. New Orleans: AIAA, 2012

4 Baumgardner D, Rodi A. Laboratory and wind tunnel evaluations of the Rosemount icing detector. J Atmosph Ocean Technol, 1989, 6(6): 971-979

5 Roy S, DeAnna R, Izad A, et al. Miniature ice detection sensor systems for aerospace applications. In: Proceedings of the 11th Annual International Workshop on MEMS. Heidelberg: IEEE, 1998. 75-80

6 Li X, Shih W Y, Vartuli J, et al. Detection of water-ice transition using a lead zirconate titanate/brass transducer. J Appl Phys, 2002, 92: 106-111

7 Zhang B H, Wang H, Wang J S, et al. A method for ice detecting based on the principle of vibration (in Chinese). Chin J Sci Instrum, 2003, 24(4): 384-386 [张滨华, 王华, 王劲松, 等. 一种基于振动原理的结冰探测方法. 仪器仪表学报, 2003, 24(4): 384-386]

8 Ye L. Ice sensor based on piezoelectric device (in Chinese). Instrum Sensors, 2001, 9: 6-8 [叶林. 基于压电器件的冰传感器. 仪表技术与传 感器, 2001, 9: 6-8]

9 Gao H, Rose J L. Ice detection and classification on an aircraft wing with ultrasonic shear horizontal guided waves. IEEE Trans Ultrason Ferroelec 
Frequ Control, 2009, 56(2): 334-344

10 Hongerholt D D, Willms G, Rose J L. Summary of results from an ultrasonic in-flight wing ice detection system. Quant Nondestruct Evaluat, 2002, 615(1): 1023-1028

11 Rose J L, 著. 何存富, 吴斌, 王秀彦, 译. 固体中的超声波. 北京: 科学出版社, 2004. 89-91

12 Zheng X M, Zhao Y Z, Shi Y W. Calculation of Lamb wave dispersion curve (in Chinese). Nondestruct Test, 2003, 25: 66-69 [郑祥明, 赵玉珍, 史耀武. Lamb波频散曲线的计算. 无损检测, 2003, 25: 66-69]

13 Yu Z H. Research on active Lamb wave health detection technology for aviation structure (in Chinese). 2007, 5: 23-29 [余振华. 面向航空结 构的主动Lamb波健康检测技术研究. 南京航空航天大学, 2007, 5: 23-29]

14 Xu Y D. Damage localization of material structure based on Lamb wave active detection technique (in Chinese). Nanjing Unive Aeron Astron, 2004, 2: 37-41 [徐颖娣. 基于Lamb波主动检测技的符合材料结构损伤定位研究. 南京航空航天大学, 2004, 2: 37-41]

15 Qiu L, Yuan S F, Wang Q. Development of Lamb wave based active structural health monitoring system (in Chinese). Piezoelec Acoustoopt, 2009, 5: 763-766 [邱雷, 袁慎芳, 王强. 基于lamb波主动结构健康监测系统的研制. 压电与声光, 2009, 5: 763-766]

\title{
Numerical and experimental study on icing detection of wind turbine blade based on ultrasonic guided wave
}

\author{
ZHU ChengXiang*, ZHANG YuLei, ZHU ChunLing \& ZHAO WeiWei \\ College of Aeronautics and Astronautics, Nanjing University of Aeronautics and Astronautics, Nanjing 210016, China
}

The numerical and experimental study on ultrasonic icing detection method based on guided wave is carried out on the surface of aluminum plate. Two piezoelectric elements are bonded to the surface of the aluminum plate, one works as a generator and the other works as a receiver, which are coupling with the ice and the aluminum plate to establish the numerical simulation model. Propagation characteristics of Lamb wave excited by the generator are calculated using numerical simulation method. Analysis of characteristic parameters of lamb wave in different frequency conditions is carried out to obtain the relationship between ice and ultrasonic wave propagation characteristics. The ultrasonic icing detection test platform is established and ultrasonic signals in the conditions of ice with different length and thickness are monitored, which are compared with that in in a clean plate of waveform. Analysis of effects of icing parameters on the guided wave propagation time and peak waveform was investigated. The results show that the peak time of ultrasonic guided wave propagation is delayed, and with the increase of the ice thickness and length, the peak time of ultrasonic guided wave propagation increases linearly.

piezoelectric, ultrasonic guided wave, ice detection, modal analysis, experiment of ice detection

PACS: 29.40.WK, 40.05.+e, 47.11.Hj, 47.50.Cd

doi: $10.1360 /$ SSPMA2016-00290 